\title{
Effect of Bisphosphonates on Anodized and Heat-Treated Titanium Surfaces: An Animal Experimental Study
}

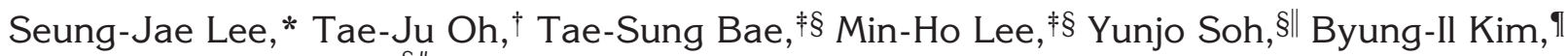
and Hyung Seop Kim*§\#

Background: Recent investigations reported that osseointegration of titanium implants can be significantly reinforced with a nanostructure treated with anodic oxidation and heat treatment. This experimental study investigates the effect of bisphosphonates on the nanotubular implant surface in rats.

Methods: Thirty-six titanium implants were divided into three groups: 1) machine-turned (MT), 2) anodized and heattreated $(\mathrm{AH})$, and 3 ) anodized and heat- and bisphosphonatetreated (AHB) groups. The 36 implants were randomly placed in both tibias of 18 male Wistar rats. After 2 and 4 weeks, the levels of osseointegration of the implants were evaluated by a removal torque test and microcomputerized tomography $(\mu \mathrm{CT})$. Peri-implant bone tissue on the extracted region was examined for the expression of type I collagen and osteocalcin.

Results: The AHB group showed the highest removal torque at 2 and 4 weeks $(13.92 \pm 1.51 \mathrm{Ncm}$ and $18.10 \pm 2.15 \mathrm{Ncm}$, respectively) followed, in order, by the AH group (11.63 \pm 1.58 $\mathrm{Ncm}$ at 2 weeks and $14.80 \pm 2.34 \mathrm{Ncm}$ at 4 weeks) and MT group (4.30 $\pm 0.76 \mathrm{Ncm}$ at 2 weeks and $6.20 \pm 1.33 \mathrm{Ncm}$ at 4 weeks) with statistically significant differences between the MT and other two groups at both time points. $\mu C T$ images also revealed a denser appearance around implants in the AHB group than in the other groups. Levels of type I collagen and osteocalcin expression were similar between the MT and AH groups; however, the values were significantly higher in the AHB group compared to the other groups, which were $220.85 \% \pm 71.09 \%$ and $363.04 \% \pm 100.21 \%$, respectively $(P<0.05)$.

Conclusion: Within the limits of this experiment, it was concluded that surface loading with bisphosphonates significantly improved the degree of osseointegration of titanium implants with a nanostructure. J Periodontol 2011;82:1035-1042.

\section{KEY WORDS}

Bisphosphonates; dental implants; nanotubes; osseointegration.

\footnotetext{
* Department of Periodontology, School of Dentistry, Chonbuk National University, Jeonju Jeonbuk, South Korea.

$\dagger$ Department of Periodontics and Oral Medicine, School of Dentistry, University of Michigan, Ann Arbor, MI.

‡ Department of Dental Biomaterials, School of Dentistry, Chonbuk National University.

$\S$ Institute of Oral Bioscience and Brain Korea 21 Project, Chonbuk National University.

|| Department of Dental Pharmacology, School of Dentistry, Chonbuk National University.

II Department of Future Plan and New Material Engineering, Sunchon National University,

Sunchon, Jeonnam, South Korea.

\# Research Institute of Clinical Medicine, Chonbuk National University.
}

$\mathrm{O}$ ver the past several decades, titanium endosseous dental implants were used as an alternative functional and esthetic restoration in fully or partially edentulous patients. ${ }^{1}$ The predictable success of endosseous dental implants is based on the primary stability in the placement of dental implants and the long-term rigid osseointegration formed between the host bone and implants. The characteristics of implant surface are an essential factor to consider when attempting to accelerate osseointegration.

Titanium has been used extensively in the field of dentistry and orthopedic surgery because of its excellent mechanical properties, higher degree of corrosion resistance, low specific weight, and bioinertness. The bioinertness of titanium results from the non-reactive titanium dioxide $\left(\mathrm{TiO}_{2}\right)$ layer, which is formed spontaneously on the surface of titanium in the body. ${ }^{2}$ This oxide layer helps prevent the degradation of dental implants in the human body. However, a prolonged period of time is needed for osseointegration compared to bioactive materials. ${ }^{3}$ The latest clinical trends of dental implants focus on methods to reduce the overall treatment period. For this reason, various surface-treatment methods to promote activity to the implant surface have been proposed. ${ }^{4,5}$

In recent years, it was reported that the formation of a titanium implant surface

doi: 10.1902/jop.2010.100608 
with a nanostructure could significantly reinforce the osseointegration. ${ }^{6-12}$ An oxide layer of nanotube titania with an ordered array, larger surface area, and higher level of surface energy can be formed by an anodic oxidation treatment on pure titanium or titanium-alloy surfaces. ${ }^{6-12}$ An anodic oxidation treatment can form an oxide film with a homogeneous thickness regardless of the surface shape of dental implants. The thickness and characteristics of the oxide layer can be adjusted by controlling processing factors, such as voltage, current density, and electrolyte compositions. ${ }^{13}$ The surface of a nanostructure can induce the migration of osteoblasts ${ }^{6-8}$ and mesenchymal stem cells ${ }^{9-11}$ and promote cell differentiation and matrix synthesis, leading to accelerated osseointegration. Because materials, such as hydroxyapatite and collagen, that form on an implant surface during osseointegration have a nanosized structure, it is believed that a nanostructured surface would be more favorable for osseointegration. ${ }^{12}$

The empty space of $\mathrm{TiO}_{2}$ nanotubes can be used as a drug-delivery carrier (i.e., as an ideal drug-storage matrix that can locally deliver drugs over a long period after the implantation of dental implants). The local delivery of drugs in dental implants aims to release the drugs solely at the specific target with a relatively lower dose and minimize the systemic drug toxicity. ${ }^{7,14}$ The drug immobilization into a nanotube can be applied to various chemicals, drugs, and biomolecules with sizes ranging from small molecules to proteins, which can reduce the inflammation and promote the osteoblastic functions, thereby accelerating osseointegration. ${ }^{14,15}$

Bisphosphonates are drugs that are most commonly prescribed to patients with osteoporosis. Bisphosphonates, which are one of the pyrophosphate analogs, are composed of a P-C-P bond binding to hydroxyapatite (HA) crystals and two lateral chains. Of the lateral chains, R1 is associated with the binding force to bone mineral, and R2 plays a role in the antiosteoclastic efficacy. If osteoclasts can absorb bisphosphonates during bone resorption, the cytoskeleton within the osteoclasts would be destroyed, resulting in a loss of the boneresorption function, which would eventually lead to an apoptosis. Moreover, this phenomenon suppresses bone resorption strongly by suppressing the differentiation of osteoclasts and inhibiting the osteoclastic activity by promoting osteoblasts. ${ }^{16}$ In vivo studies ${ }^{17-19}$ of dental implants, whose surfaces were locally coated with bisphosphonates, revealed larger amounts of bone formation and a higher degree of bone-to-implant contact in the early stage than on non-coated implants. Therefore, the use of bisphosphonates on the surface of dental implants was believed to promote osteogenesis and to achieve faster osseointegration by suppressing the activity of osteoclasts on the interface between the bone tissue and dental implants.
Although positive effects of the nanotube structure or bisphosphonates on the osseointegration of titanium implants were suggested, ${ }^{6-12,17-19}$ there is a lack of up-to-date information regarding the effect of bisphosphonates on the osseointegration of nanotubular implants. Therefore, the purpose of this animal study was to investigate additional effects of bisphosphonates on the osseointegration of anodized and heat-treated $(\mathrm{AH})$ titanium surfaces.

\section{MATERIALS AND METHODS}

\section{Preparation of Samples With a Nanotube $\mathrm{TiO}_{2}$ Layer, Heat, and Ibandronate Treatment}

Thirty-six dental implants $(2-\mathrm{mm}$ diameter $\times 4-\mathrm{mm}$ length with five to six screw threads) were manufactured from commercially pure grade IV titanium** in a customizable manner. The dental implants were rinsed ultrasonically in a solution containing acetone and alcohol for 5 minutes each. All implants were stored in a dryer at $50^{\circ} \mathrm{C}$ for more than 24 hours before the anodic oxidation treatment. To form $\mathrm{TiO}_{2}$ nanotubes, a titanium implant and platinum plate were connected to the cathode and anode of a direct-current power generator, ${ }^{\dagger \dagger}$ respectively, and a pulse power of $20 \mathrm{~V}$ and $20 \mathrm{~mA} / \mathrm{cm}^{2}$ in 0.01 -millisecond cycles was applied for 60 minutes. The electrolyte solutions were prepared by mixing glycerol with 20 weight \% (wt\%) $\mathrm{H}_{2} \mathrm{O}$ and 1 wt\% $\mathrm{NH}_{4} \mathrm{~F}$. After the anodic oxidation treatment, the sample surface was rinsed ultrasonically for 1 minute. The samples were stored in a dryer at $50^{\circ} \mathrm{C}$ for 24 hours. Topographic characteristics of the $\mathrm{TiO}_{2}$ nanotube structure were observed under field emission-scanning electron microscopy (FE-SEM). ${ }^{\neq}$The structural stability of the nanotube $\mathrm{TiO}_{2}$ layer and the removal of impurities were achieved by placing the samples in a furnace heated to $500^{\circ} \mathrm{C}$ at a heating rate of $10^{\circ} \mathrm{C} /$ minute for 2 hours. For bisphosphonate treatment, one group of the antiosteoporotic agents, ibandronate, was diluted in distilled water at a concentration of $1 \mathrm{mg} / \mathrm{mL}$. Each implant was immersed in the diluted drugs in a $37^{\circ} \mathrm{C}$ incubator for 24 hours to allow for the absorption of the drug.

\section{Experimental Design and Implantation Procedure} The study was conducted in compliance with the principles of the Helsinki Declaration of 1975, as revised in 2000. Ethical clearance of the study was approved by the Institutional Animal Care and Use Committee of the Chonbuk National University Laboratory Animal Center, Jeonju, South Korea.

For the experiment, both tibias of 18 male Wistar rats (12 weeks old and weighing 200 to $225 \mathrm{~g}$ ) were

\footnotetext{
** Kobe Steel, Tokyo, Japan.

$\dagger \dagger$ Kwangduck, Bucheon, South Korea.

キ S-800, Hitachi, Tokyo, Japan.
} 
used (total $N=36$ ). Thirty-six dental implants were divided into the following three groups: 1) machineturned (MT) (untreated titanium; $\mathrm{n}=12), 2) \mathrm{AH}(\mathrm{n}=$ 12 ), and 3 ) anodized and heat- and bisphosphonatetreated (AHB) (implants treated with ibandronate immobilization after the anodic oxidation and thermal treatment; $\mathrm{n}=12$ ).

The rats were allowed to acclimatize for $\approx 1$ week before the experimental study. The implant placement surgery was performed under general anesthesia using intramuscular administration of $1: 1$ mixture of tiletamine and zolazepam $(150 \mathrm{uL} / 200 \mathrm{~g}) . \S \S$ The surgical site was shaved and disinfected with a betadine scrub. An incision of $1.0 \mathrm{~cm}$ in length was made, and the bone surface of the tibiae was surgically exposed by blunt dissection. Under a profuse saline-solution irrigation, bicortical implant beds were drilled at a rotary speed $\leq 1,500 \mathrm{rpm}$. In total, 36 screw-shaped titanium implants were placed using a self-tapping process in the medial region of the bilateral tibia diaphysis in the 18 rats until the screw thread was completely introduced into the bone cortex. Resorbable sutures $\|||$ were used to approximate the surgical wound. Postoperatively, amoxicillin $(6 \mathrm{mg} / \mathrm{kg})$ antibiotics and nabumetone $(5 \mathrm{mg} / \mathrm{kg})$ non-steroidal anti-inflammatory drugs were orally administered by dissolving them in the drinking water. Nine rats, each at 2 and 4 weeks after implantation, were sacrificed with an overdose of thiopental|fI (six implants per time point, per group).

\section{Removal Torque Analysis}

The removal torque was measured at 2 and 4 weeks post-implantation. Implant sites in the rat tibia were surgically exposed via a sharp dissection to bone and clinically examined after the careful removal of overgrowing bone and soft tissues. An implant removal mount was securely fastened and engaged the external hex, which was connected to the torque gauge with the insertion device. Removal torque tests were performed on all implants (six implants per time point, per surface) in the tibia using a digital torque gauge. ${ }^{\# \#}$ After stabilizing the legs, the torque was increased incrementally by slowly rotating the gauge counterclockwise. The manual torquing continued until a loosening of the implants was detected with the peak torque value recorded when a rupture occurred between the implant and bone. Peak torque measurements were recorded by a single examiner (T-SB) who was masked to the implant surface characteristics. Mean torque measurements were calculated for each group.

\section{Analysis of Bone-Formation Markers by Western Blot}

At 4 weeks, immediately after removal torque measurements, bone tissue was collected in the areas adjacent to the surface of the implants that were removed. The tissue sample (six implants per surface) was rinsed with saline, and the blood components were removed. The tissue sample was ground using a homogenizer after adding a protein lysis buffer (20 mM Tris- $\mathrm{HCl}$ [pH 7.5], $137 \mathrm{mM} \mathrm{NaCl}, 10 \%$ glycerol, $1 \%$ Triton X-100, $1 \mathrm{mM} \mathrm{Na}_{3} \mathrm{VO}_{4}, 1 \mathrm{mM}$ phenylmethylsulfonylfluoride, and $1 \times$ protease inhibitor cocktail). After centrifugation at $12,000 \times g$ for 15 minutes, supernatants were used as cell extracts. Levels of type I collagen and osteocalcin expression, which were involved in the maturation and mineralization of osteocytes, were confirmed by western blotting with appropriate antibodies. Total proteins from the rat tibia were separated with $10 \%$ sodium dodecyl sulfate-polyacrylamide gel electrophoresis and transferred to a polyvinylidene difluoride membrane. ${ }^{* *}$ Membranes were blocked with 5\% non-fat skim milk (weight/volume) in Tris-buffered saline containing 0.5\% Tween20 (volume/volume) (TTBS) at room temperature for 1 hour and then incubated overnight at $4^{\circ} \mathrm{C}$ with rabbit anti-type I collagen ${ }^{\dagger \dagger}$ or antiosteocalcin ${ }^{\ddagger \dagger}$ antibodies diluted $1: 1,000$ in 5\% non-fat skim milk in TTBS. A horseradish peroxidase-conjugated antirabbit antibody $\S \S \S$ was used as the secondary antibody (1:5,000 to $1: 10,000$ dilution in $5 \%$ non-fat skim milk in TTBS for a 1-hour incubation at room temperature), and the antigen-antibody complexes were visualized using a kit.. $\mid\|1\|$

\section{Microcomputerized Tomography $(\mu \mathrm{CT})$ Observation}

One day before sacrifice at 2 and 4 weeks, the experimental rats were anesthetized, and implant areas were scanned with a $\mu \mathrm{CT}$ scanner. . $\mid$ tा The voltage and current of the $\mathrm{x}$-ray tube were $100 \mathrm{kV}$ and 100 $\mu \mathrm{A}$, respectively, with 240 milliseconds of exposure time. X-ray projections were obtained at $0.6^{\circ}$ intervals with a scanning angular rotation of $360^{\circ}$. The image pixel size was set for $18.104 \mu \mathrm{m}$. Among those microimages, we especially selected images in which a screw line could be observed with the cross-section following the long axis of the implant.

\section{Statistical Analyses}

The mean and SD of removal torque values and protein expression were calculated. Independent $t$ tests were used to compute 1) $P$ values for differences in removal torque values of each group between 2 and

\footnotetext{
$\S \S$ Zoletil 50, Virbac Laboratories, Carros, France.

||| 4.0 Polyglactin 910 (Vicryl), Ethicon, Livingston, U.K.

II Thiopental sodium, ChoongWae Pharma, Seoul, South Korea.

\#\# 98109, Aikoh Engineering, Osaka, Japan.

*** Bio-Rad, Hercules, CA.

$\dagger \dagger \dagger$ Santa Cruz Biotechnology, Santa Cruz, CA.

玤 Santa Cruz Biotechnology.

$\S \S \S$ Santa Cruz Biotechnology.

I\|l\| ECL Plus, Amersham Biosciences, Piscataway, NJ.

ๆๆๆ Skyscan1076, Skyscan, Aartselaar, Belgium.
} 
4 weeks, and 2) differences in the expression of bone-formation markers (type I collagen and osteocalcin) between the MT group and other groups (AH and AHB groups). The Tukey test was used to compute $P$ values for differences in removal torque values among the three groups (MT, $\mathrm{AH}$, and AHB). $P<0.05$ was considered statistically significant. All statistical analyses were carried out with computer software. $\# \# \#$

\section{RESULTS}

\section{Surface Structure of the Nanotube $\mathrm{TiO}_{2}$ Layer of Dental Implants}

Figure 1 shows FE-SEM images of the implant surface after anodizing treatment. As shown in Figure 1C, the surface consisted of a dense structure of independent, self-organized nanotubes with mean diameters of $152.6 \pm 16.2 \mathrm{~nm}$ and $80.3 \pm 10.8 \mathrm{~nm}$ (Fig. 1C). Cross-sectional lateral views of the layer were obtained and showed that the diameters of nanotubes increased from the superior to inferior direction with a mean length of $506.7 \pm 25.0 \mathrm{~nm}$ (Fig. 1D). The nanotubes that were sectioned longitudinally revealed the presence of multiple empty spaces (Fig. 1E).

\section{Removal Torque Analysis}

Table 1 presents the removal torque measured at 2 and 4 weeks after placing the dental implants. The removal torque was higher at 4 weeks than at 2 weeks in all groups, with the highest values in the AHB group at both time points (Table 1 ). In addition, statistically significant differences were found among the MT and the other two groups at both time points, with the MT being lowest $(P<0.05)$ (Fig. 2).

\section{Analysis of Bone Formation Markers}

At 4 weeks postimplantation, the degree of mineralization was evaluated based on the levels of type I collagen and osteocalcin expression by western blot analysis. Levels of type I collagen and osteocalcin expression were similar in the bone tissues from the MT and AH groups. In contrast, there were significantly higher levels of type I collagen and osteocalcin expression in the bone tissue from the AHB group compared to other groups. Especially when compared with the MT group (100\%) the collagen expression level of the AHB group was about two times higher $(220.85 \% \pm 71.09 \%)$ and the osteocalcin expression was about 3.6 times higher (363.04\% $\pm 100.21 \%)$ than the MT group.

\section{$\mu \mathrm{CT}$ Observation}

Figure 4 shows $\mu C T$ images of implants placed in rat tibiae at 2 and 4 weeks after implantation. In the MT group, only the outer area of threads was radiopaque, with the inner area of threads being radiolucent on the images. The AH group also exhibited a similar finding. However, it was remarkable to see the AHB group displaying radiopacity on the outer and inner areas of threads. This implied that the intimate contacts between the implant and surrounding bone in the AHB group were the result of more bone deposition on the implant surface. This feature was more remarkable at 4 weeks than at 2 weeks, and the overall bone density also increased at 4 weeks.

\section{DISCUSSION}

For the present study, the test titanium implants underwent anodic oxidation to form a nanotube, followed by a thermal treatment. Subsequently, ibandronate, one of the bisphosphonates, was penetrated into the nanotube structure. The results of this study revealed that the AHB implant group showed excellent biocompatibility and osseointegration that were superior to the MT and AH groups. This superiority was well demonstrated by the highest values of removal torque, protein expression, and analyses.

The removal torque analysis at 2 and 4 weeks revealed the highest values found in the AHB group as shown in Table 1 and Figure 2. In addition, in all groups, the removal torque was relatively higher at 4 weeks than at 2 weeks, with statistically significant differences found in the MT and AHB groups. These outcomes correspond well with results of other studies ${ }^{20,21}$ in which bisphosphonate-treated titanium implants displayed a higher mechanical fixation than uncoated titanium implants. However, the experimental method used in the previous studies was different from our method. Although, in the present study, bisphosphonate treatment was made through nanotubes on the implant surface, the other studies used bisphosphonate treatment on the surface of hydoxyapatite-coated titanium implants. Furthermore, the results showed that the $\mathrm{AH}$ and AHB groups had significantly higher values of removal torque than did the MT group (Fig. 2). This result showed that the rough surface with a $\mathrm{TiO}_{2}$ nanotube layer might have contributed to the increased values of removal torque in the $\mathrm{AH}$ and AHB groups. The most-increased removal torque values in the AHB group indicated a higher friction force between the implant and surrounding bone, suggesting that a higher degree of osseointegration existed in this type of implant.

$\mu \mathrm{CT}$ images taken at 2 and 4 weeks after placing dental implants showed that the areas adjacent to the implant screws were clearer and denser in the AHB group than in the MT and AH groups (Fig. 4). This result indicated that the degree of bone contact with the implants was relatively higher in the AHB group, and more bone was formed in the areas adjacent to the implant. The $\mu \mathrm{CT}$ results and increased removal torque suggested that the surface treated with bisphosphonates

\#\#\# SPSS 12.0 for Windows, IBM, Chicago, IL. 
as well as the surface with a nanotube structure might have induced an increase in bone formation, which could have affected the physiochemical interactions with adjacent bones.

The increased expression of bone formation markers (Fig. 3) indirectly reflect the increased bone formation in the AHB group. The degree of type I collagen and osteocalcin expression was highest in the AHB group and with statistical significance. Type I collagen is one of the most common collagenous bone proteins that accounts for $>90 \%$ of the bone matrix, which reflects the bone formation. Osteocalcin is an osteogenic marker that reflects the functions of osteoblasts in association with the minerals contained in the bone matrix. Hence, the highest levels of type I collagen and osteocalcin expression in the AHB group indicated that the activities of bone formation were the greatest in the bisphosphonate-treated nanotubular implants.

Positive effects of bisphosphonate treatment on the implant surface in promoting osseointegration were demonstrated in the literature. ${ }^{17-19,22}$ Meraw et al. ${ }^{17,18}$ examined the effects of alendronate on bone regeneration after an alendronate treatment on the surface of dental implants in an animal experimental model using dogs. They ${ }^{17,18}$ reported that the use of alendronate increased the initial bone formation and bone-to-implant contact in the areas adjacent to the implant. ${ }^{17,18}$ Zuffetti et al. ${ }^{19}$ performed a histopathologic examination on bisphosphonate-treated implants 2 months post-implantation in humans and showed that the bone density and degree of bone-to-implant contact were higher in bisphosphonate-treated implants than in control implants. Furthermore, in a recent report, ${ }^{22}$ bisphosphonate-treated implants were placed in the maxilla of five patients using a two-stage surgical procedure, and the implant stability quotient (ISQ) was measured at stage-I implant surgery and after 6 months. The results revealed that the largest improvements in the ISQ level of all implants were found for bisphosphonate-treated implants.

There is still some controversy regarding the mechanisms by which $\mathrm{TiO}_{2}$ nanotubes are formed by anodic oxidation. The formation of nanotubes has been suggested to originate from the electrochemical growth of an oxide layer and the dissolution

Table I.

Removal Torque Values at 2 and 4 Weeks (six implants per time point per surface)

\begin{tabular}{lccc}
\hline Removal Torque & MT Group* & AH Group & AHB Group* \\
\hline 2 weeks (Ncm; mean $\pm \mathrm{SD})$ & $4.30 \pm 0.76$ & $11.63 \pm 1.58$ & $13.92 \pm 1.51$ \\
4 weeks $(\mathrm{Ncm}$; mean $\pm \mathrm{SD})$ & $6.20 \pm 1.33$ & $14.80 \pm 2.34$ & $18.10 \pm 2.15$ \\
\hline
\end{tabular}

* MT and AHB groups showed significant differences between the 2- and 4-week time points $(P<0.05$; independent $t$ test). 


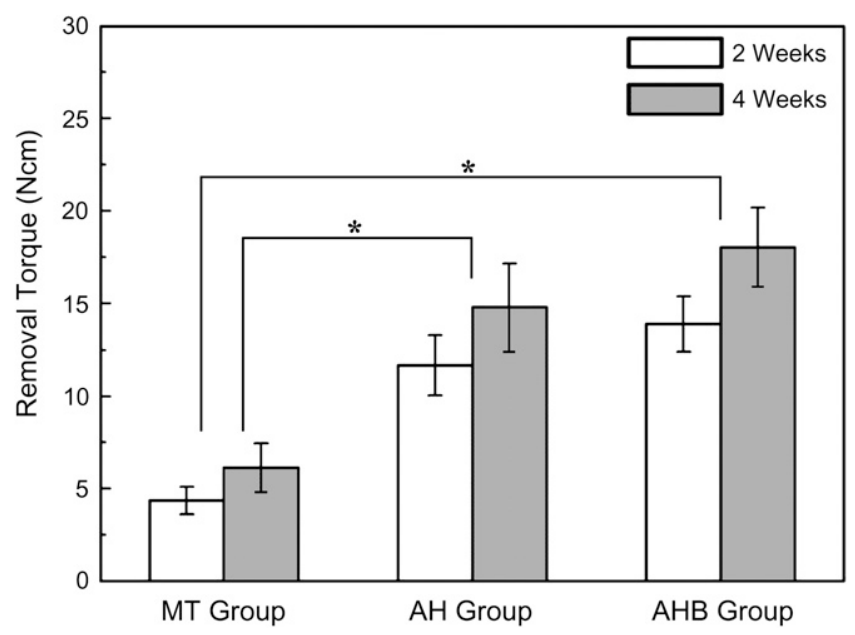

Figure 2.

Comparison of removal torque values among groups. *Statistically significant differences between the MT group and $\mathrm{AH}$ and $\mathrm{AHB}$ groups at 2 and 4 weeks ( $P<0.05$; Tukey test). chemical oxidation. Subsequently, the oxide layer that formed in the early stage dissolved, followed by the formation of a self-organized $\mathrm{TiO}_{2}$ nanotube layer after 1 hour of anodic oxidation.

The empty space within the $\mathrm{TiO}_{2}$ nanotube can be used as a drug-delivery carrier that can reduce the inflammation or accelerate osseointegration by promoting osteoblastic functions as well as by suppressing osteoclast activities. According to Yao and Webster, ${ }^{14}$ penicillin loaded into the anodized titanium with a nanotube structure using a coprecipitation method in vitro was released over a 3-week period via first-order kinetics. Compared to the untreated or anodized titanium, the level of bone-cell adhesion was further increased. ${ }^{14}$ In addition, according to Bae et al., ${ }^{15}$ after loading the immobilized recombinant human bone morphogenetic protein 2 in vitro, the drugs were released gradually from the anodized titanium with a nanotube structure over a 3-week period. The activity of osteoblasts was relatively higher than that observed on the nonanodized titanium samples. ${ }^{15}$ Our previous in vitro study on the release mechanism of ibandronate also revealed that the drug deposition and release activity were considerably improved with the nanotube structure (unpublished data). In the present study, the controlled release of bisphosphonates might have continuously promoted the osseointegration of the nanotube implants during the healing period as demonstrated by the higher mean removal torque values in the AHB group than in the MT and AH groups.

Bisphosphonates are known to have a strong affinity to calcium and $\mathrm{HA}$, which have a great impact on the bone metabolism by binding to the bone tissue. Currently, bisphosphonates are used to treat bone metabolic diseases, such as osteoporosis, Paget disease, multiple myeloma, and solid tumors associated with the bone metabolism. Reddy et al. ${ }^{24}$ and Weinreb et al. ${ }^{25}$ investigated the effects of bisphosphonates in animal models in which alveolar bone resorption was induced by experimental periodontitis. In the studies, ${ }^{24,25}$ it was shown that the systemic administration of alendronate showed a positive effect in 

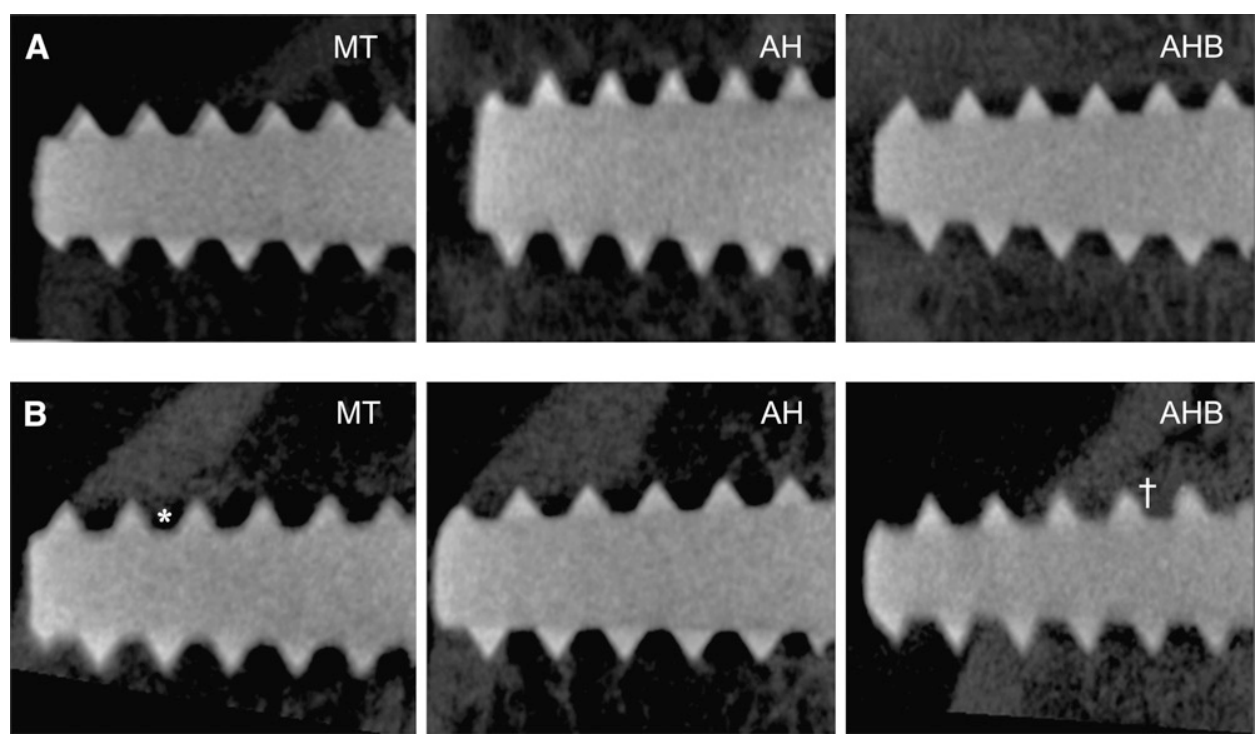

\section{Figure 4.}

$\mu C T$ scan images at 2 (A) and 4 (B) weeks. *The bottom area of threads of the MT implant was radiolucent, which meant the absence of bone. "The bottom area of threads of the AHB implant was radiopaque indicating the presence of bone.

reducing bone loss and increasing bone density. In other studies, ${ }^{26,27}$ dental implants were placed with or without a subcutaneous injection of alendronate in rats in which osteoporosis was induced by a bilateral ovariectomy. Removal torque values were significantly higher in the alendronate-treated group than in the other group that had undergone an ovariectomy only. The results of the studies ${ }^{26,27}$ suggested that systemic bisphosphonate administration might have improved the osseointegration of titanium implants placed in osteoporotic bone.

The systemic use of bisphosphonates has been cautioned because of some side effects, such as esophagitis, gastric ulcers, and osteonecrosis of the jaw (ONJ). ${ }^{28-31}$ In particular, ONJ may occur after a tooth extraction (or a treatment involving the dento-alveolar structure) in a patient who received a systemic administration of bisphosphonates. ONJ was first reported in $2003,{ }^{28}$ but the precise mechanism is unclear. According to Marx et al., ${ }^{29}$ the occurrence of ONJ might be associated with an impairment of bone remodeling and regeneration and the insufficient blood supply in patients given bisphosphonates. More than $90 \%$ of reported cases were described in cancer patients who received an intravenous injection of bisphosphonate at higher doses. In these cases, the incidence was associated with the dose and treatment period. According to the latest research, ${ }^{30,31}$ only the use of a high dose of bisphosphonates was associated with the occurrence of ONJ in cancer patients. However, it was reported that the use of a lower bisphosphonate dose might have almost no relationship with the occurrence of ONJ in patients with osteoporosis. ${ }^{30,31}$ Therefore, it was suggested that the local use of bisphosphonates in dental implants may not be contraindicated. ${ }^{30,31}$

\section{CONCLUSIONS}

In this in vivo, non-human experiment, the removal torque, bone density, and degree of bone-formation marker expression increased after an ibandronate treatment of an anodized implant with a nanotube structure. These results suggested that this surface treatment increased the biocompatibility of the implant surface, promoted the formation of the adjacent bone, and accelerated osseointegration. Additional studies, including long-term clinical trials and histologic and histomorphometric analyses, are required to confirm the results of the present study.

\section{ACKNOWLEDGMENTS}

This article was supported by the Chonbuk National University Hospital Research Institute of Clinical Medicine, Jeonju, South Korea, and by a Korea Science and Engineering Foundation grant (R01-2008-000-20556-0) funded by the Korea Ministry of Education, Science and Technology (MEST), Seoul, South Korea. The authors report no conflicts of interest related to this study.

\section{REFERENCES}

1. Pjetursson BE, Karoussis I, Bürgin W, Brägger U, Lang NP. Patients' satisfaction following implant therapy. A 10-year prospective cohort study. Clin Oral Implants Res 2005;16:185-193.

2. Textor M, Sittig C, Frauchiger V, Tosatti S, Brunnete DM. Properties and biological significance of natural oxide films on titanium and its alloys. In: Brunette DM, Tengvall $\mathrm{P}$, Textor $\mathrm{M}$, Thomsen $\mathrm{P}$, eds. Titanium in Medicine. Berlin: Springer-Verlag; 2001:171-230.

3. Ellingsen JE, Johansson CB, Wennerberg A, Holmen A. Improved retention and bone implant contact with fluoride-modified titanium implant. J Oral Maxillofac Implants 2004; 19:659-666.

4. Albrektsson T, Wennerberg A. Oral implant surfaces: Part 1 - Review focusing on topographic and chemical properties of different surfaces and in vivo responses to them. Int $J$ Prosthodont 2004; 17:536-543.

5. Albrektsson T, Wennerberg A. Oral implant surfaces: Part 2 - Review focusing on clinical knowledge of different surfaces. Int J Prosthodont 2004;17:544-564. 
6. Brammer KS, Oh S, Cobb CJ, Bjursten LM, van der Heyde H, Jin S. Improved bone-forming functionality on diameter-controlled $\mathrm{TiO}_{2}$ ) nanotube surface. Acta Biomater 2009;5:3215-3223.

7. Popat KC, Eltgroth M, Latempa TJ, Grimes CA, Desai TA. Decreased Staphylococcus epidermis adhesion and increased osteoblast functionality on antibiotic-loaded titania nanotubes. Biomaterials 2007;28:4880-4888.

8. Balasundaram G, Yao C, Webster $\mathrm{TJ} . \mathrm{TiO}_{2}$ nanotubes functionalized with regions of bone morphogenetic protein-2 increases osteoblast adhesion. $J$ Biomed Mater Res A 2008;84:447-453.

9. Popat KC, Leoni L, Grimes CA, Desai TA. Influence of engineered titania nanotubular surfaces on bone cells. Biomaterials 2007;28:3188-3197.

10. Oh S, Brammer KS, Li YS, et al. Stem cell fate dictated solely by altered nanotube dimension. Proc Natl Acad Sci USA 2009;106:2130-2135.

11. Park J, Bauer S, Schlegel KA, Neukam FW, von der Mark K, Schmuki P. $\mathrm{TiO}_{2}$ nanotube surfaces: $15 \mathrm{~nm}-$ An optimal length scale of surface topography for cell adhesion and differentiation. Small 2009;5:666-671.

12. Bjursten LM, Rasmusson L, Oh S, Smith GC, Brammer KS, Jin S. Titanium dioxide nanotubes enhance bone bonding in vivo. J Biomed Mater Res A 2010;92:1218-1224.

13. Ishizawa $\mathrm{H}$, Ogino $M$. Formation and characterization of anodic titanium oxide films containing $\mathrm{Ca}$ and $\mathrm{P}$. $J$ Biomed Mater Res 1995;29:65-72.

14. Yao C, Webster TJ. Prolonged antibiotic delivery from anodized nanotubular titanium using a co-precipitation drug loading method. J Biomed Mater Res B Appl Biomater 2009;91:587-595.

15. Bae IH, Yun KD, Kim HS, et al. Anodic oxidized nanotubular titanium implants enhance bone morphogenetic protein-2 delivery. J Biomed Mater Res B Appl Biomater 2010;93:484-491.

16. Moreau MF, Guillet C, Massin P, et al. Comparative effects of five bisphosphonates on apoptosis of macrophage cells in vitro. Biochem Pharmacol 2007;73:718-723.

17. Meraw SJ, Reeve CM, Wollan PC. Use of alendronate in peri-implant defect regeneration. J Periodontol 1999; 70:151-158.

18. Meraw SJ, Reeve CM. Qualitative analysis of peripheral peri-implant bone and influence of alendronate sodium on early bone regeneration. J Periodontol 1999; 70:1228-1233.

19. Zuffetti F, Bianchi F, Volpi R, et al. Clinical application of bisphosphonates in implant dentistry: Histomorphometric evaluation. Int $J$ Periodontics Restorative Dent 2009;29:31-39.

20. Peter B, Gauthier O, Laïb S, et al. Local delivery of bisphosphonate from coated orthopedic implants increases implants mechanical stability in osteoporotic rats. J Biomed Mater Res A 2006;76:133-143.
21. Gao Y, Zou S, Liu X, Bao C, Hu J. The effect of surface immobilized bisphosphonates on the fixation of hydroxyapatite-coated titanium implants in ovariectomized rats. Biomaterials 2009;30:1790-1796.

22. Abtahi J, Tengvall P, Aspenberg P. Bisphosphonate coating might improve fixation of dental implants in the maxilla: A pilot study. Int J Oral Maxillofac Surg 2010;39:673-677.

23. Crawford GA, Chawla N, Das K, Bose S, Bandyopadhyay A. Microstructure and deformation behavior of biocompatible $\mathrm{TiO}_{2}$ nanotubes on titanium substrate. Acta Biomater 2007;3:359-367.

24. Reddy MS, Weatherford TW 3rd, Smith CA, West BD, Jeffcoat MK, Jacks TM. Alendronate treatment of naturally-occurring periodontitis in beagle dogs. J Periodontol 1995;66:211-217.

25. Weinreb M, Quartuccio H, Seedor JG, et al. Histomorphometrical analysis of the effects of the bisphosphonate alendronate on bone loss caused by experimental periodontitis in monkeys. J Periodontal Res 1994;29: 35-40.

26. Giro G, Sakakura CE, Gonçalves D, Pereira RM, Marcantonio E Jr., Orrico SR. Effect of 17beta-estradiol and alendronate on the removal torque of osseointegrated titanium implants in ovariectomized rats. J Periodontol 2007;78:1316-1321.

27. Narai S, Nagahata S. Effects of alendronate on the removal torque of implants in rats with induced osteoporosis. Int J Oral Maxillofac Implants 2003;18:218223.

28. Marx RE. Pamidronate (Aredia) and zoledronate (Zometa) induced avascular necrosis of the jaws: A growing epidemic. J Oral Maxillofac Surg 2003;61:1115-1117.

29. Marx RE, Sawatari Y, Fortin M, Broumand V. Bisphosphonate-induced exposed bone (osteonecrosis/ osteopetrosis) of the jaws: Risk factors, recognition, prevention, and treatment. J Oral Maxillofac Surg 2005;63:1567-1575.

30. Lo JC, O'Ryan FS, Gordon NP, et al. Prevalence of osteonecrosis of the jaw in patients with oral bisphosphonate exposure. J Oral Maxillofac Surg 2010;68:243253.

31. Khan AA, Sándor GK, Dore E, et al. Bisphosphonate associated osteonecrosis of the jaw. J Rheumatol 2009;36:478-490.

Correspondence: Dr. Hyung Seop Kim, Department of Periodontology, Chonbuk National University School of Dentistry, 634-18 Guman-dong, Dukjin-gu, Jeonju, Korea 561-712. Fax: 82-63-250-2259; r-mail: cbuperio@jbnu.ac.kr.

Submitted October 6, 2010; accepted for publication November 24, 2010. 